OPEN ACCESS

Edited by:

Songyan Li,

China University of Petroleum

(Huadong), China

Reviewed by:

Zhengming Xu,

China University of Geosciences,

China

Zhi Ye,

University of Oklahoma,

United States

*Correspondence:

Huijun Zhao

cczuzhj.geo@foxmail.com

Specialty section:

This article was submitted to Advanced Clean Fuel Technologies, a section of the journal Frontiers in Energy Research

Received: 24 August 2020 Accepted: 30 September 2020 Published: 12 November 2020

Citation:

Deng S, Ling D, Zhao H, Shen X, Du H and Liu L (2020) Optimization Simulation Research on Middle-Deep Geothermal Recharge Wells Based on Optimal Recharge Efficiency. Front. Energy Res. 8:598229. doi: 10.3389/fenrg.2020.598229

\section{Optimization Simulation Research on Middle-Deep Geothermal Recharge Wells Based on Optimal Recharge Efficiency}

\author{
Song Deng ${ }^{1}$, Dingkun Ling ${ }^{1}$, Huijun Zhao ${ }^{1 *}$, Xin Shen ${ }^{1}$, Huajun $\mathrm{Du}^{2}$ and Lu Liu ${ }^{1}$ \\ ${ }^{1}$ School of Petroleum Engineering, Changzhou University, Changzhou, China, ${ }^{2}$ Shandong Ruiheng Xingyu Petroleum Technology \\ Development Co. Ltd, Qingdao, China
}

In order to solve the low recharge efficiency of middle and deep geothermal Wells, a thermo-hydro-mechanical (THM) model integrating geothermal production and reinjection was established, and the relationship model between recharge efficiency and production well temperature was built to obtain the optimal recharge efficiency. In view of the different well spacing between reinjection and production wells, the different well spacing between production wells and different reinjection well types, numerical simulation and comparative analysis were carried out under the optimal recharge efficiency. The results show that under the optimal recharge efficiency, the change in well spacing has the greatest impact on the exploitation of production wells. The well spacing of production wells is gradually reduced from $600 \mathrm{~m}$ to $20 \mathrm{~m}$, and the time of thermal breakthrough in production wells is getting earlier and earlier. However, when the spacing between the production wells reach $200 \mathrm{~m}$, thermal breakthrough hardly occurs. Compared with different well spacings, different reinjection well types have less impact on the exploitation of production wells, and the situation of production wells under one reinjection and two production is better than one reinjection and one production. Therefore, it is proposed that under the optimal recharge efficiency of $40.41-72.52 \%$, the most suitable approach is to use one reinjection and two production as this well spacing between the reinjection and production well above $400 \mathrm{~m}$, the well spacing between the production wells above $200 \mathrm{~m}$ and the reinjection wells of horizontal wells are optimal and most suitable for geothermal exploitation.

Keywords: Recharge efficiency, Well spacing, Thermal breakthrough, Reinjection well type, One reinjection and two production

\section{INTRODUCTION}

In recent years, the decrease of traditional fossil energy storage and the environmental pollution that accompanies it has attracted widespread attention. The harmful substances produced by these traditional energy consumption processes are far more serious than expected (Mei et al., 2018; Shen et al., 2016; Kong et al., 2017). Renewable energy and geothermal energy could be widely used in many aspects such as heating, tourism and aquaculture by virtue of large energy potentials, sustainability, and convenient development and utilization (Gao, 2018; Xu, 2018). Geothermal resources can be divided into three types: bedrock fissure thermal storage, solution fissure thermal storage and sandstone pore thermal storage according to different thermal storage media and 
recharge performance (Olasolo et al., 2016). Among them, unconsolidated sandstone reservoirs have a poor degree of rock cementation. During the development process, some problems may occur such as sand-carrying sand production and low recharge efficiency (Sedghi and Zhan, 2020). It is, therefore, necessary to study optimized designs for reinjection wells to improve the recharge efficiency of sandstone geothermal reservoirs. Reinjection is an effective protective measure: first, it can effectively treat the wastewater produced by geothermal heat, and to a certain extent, can avoid thermal pollution or pollution to the environment; second, it can help the geothermal reservoir to recover heat production and effectively improve the utilization rate of geothermal resources; third, it can maintain the pressure of geothermal reservoirs, effectively stabilizing the mining conditions of geothermal resources, and preventing ground subsidence (Liu, 2003).

Scholars all over the world have researched the optimal design for the layout of reinjection wells. For example, Yan Fang Ping compared the recharge efficiency under two different recharge modes, and different reinjection layout schemes according to the results of a simulation (Yan, 2020). Sun Jian ping and Xu Rui jian used different recharge arrangements to conduct single-well recharge tests for confined aquifers in a certain area (Sun et al., 2020). CHÁVEZ Oscar and GODÍNEZ Francisco performed numerical simulations on the thermal behavior of Mexico's geothermal reservoirs, calculated the optimal reinjection-production well distance and a variety of different configuration methods (Chávez and Godínez, 2020). In order to reduce the probability of thermal breakthrough in production wells, Liu G and Wang G proposed the arrangement of clustered wells, and the results showed that the clustered well pattern would have better results (Liu et al.,2020). Alexandros Daniilidisa analyzed the influence of physical parameters, design parameters and operating parameters on the productivity performance of the geothermal system, and summarized the best parameter settings (Daniilidis et al., 2020). Zhu Jialing studied the influence of the pressure difference between geothermal reinjection wells on the recharge efficiency, and proposed guidelines on the appropriate spacing of the extraction and reinjection wells for porous thermal storage (Zhu et al., 2012).

Most of them study the layout of reinjection wells aimed to improve the utilization rate of geothermal resources or the heat recovery rate, but research on the optimal design of the reinjection well layout based on the optimal recharge efficiency has rarely been studied. The numerical simulation of geothermal production and recharge involves multiple processes, including the heat transfer process between geothermal water, the thermal reservoir and wellbore, the heat transfer process between the wellbore and thermal reservoir, and the flow process of geothermal water and thermal reservoir and wellbore, etc. These require multiphysics coupling but these studied rarely involve multiphysics coupling. COMSOL users are more inclined to study the impact of fractures on geothermal production. There is less researches on the optimization design of well type and well spacing for the efficiency of middle-deep geothermal recharge. In response, this paper established a THM multi-field coupling model for medium and deep geothermal recovery and irrigation. According to the fitting equation of the recharge efficiency and the temperature of the production well, an optimal recharge efficiency range was obtained. Using COMSOL software, the geothermal recovery was performed under the optimal recharge efficiency. The layout of the reinjection wells was simulated, and the well type, well spacing and well layout method were optimized to provide guidance for solving the problem of low recharge efficiency of middle-deep sandstone thermal storage.

\section{GEOTHERMAL MULTI-FIELD COUPLING MODEL ESTABLISHMENT}

\section{Hypothesis Conditions}

Based on sandstone fractured thermal reservoirs, this paper establishes a THM model for reservoir reconstruction. The reservoir is divided into three layers with different hydraulic and thermal properties. The sandstone fractured thermal reservoir is simplified as a rock block-fracture dual medium. The rock block is used as a porous medium with low permeability, and the physical property changes of the rock block are not considered. The fracture can also be used as the main flow channel of geothermal water. Assuming that the water in the hydrothermal reservoir is saturated with single-phase water, the flow of water is laminar, which conforms to Darcy's law. The geothermal water in the cracks produces heat exchange and transfer by convection and conduction, which conforms to Fourier's law. It is Assumed that the temperature difference between the solid and liquid phases is small, that it is locally in a state of thermal equilibrium that gravity, the capillary force has no effect on the flow, and that the fluid does not react chemically with the rock mass.

\section{Governing Equation}

According to the theory of THM, the seepage and heat transfer in the model are described, including the seepage field, temperature field equation and water flow continuity equation, as shown below:

(1) Seepage field equation

The seepage field equation of the rock:

$$
\begin{gathered}
S \frac{\partial p}{\partial t}+\nabla \cdot u=-\frac{\partial e}{\partial t}+Q \\
\mathrm{u}=-\frac{\kappa}{\eta}(\nabla p+\rho g \nabla z)
\end{gathered}
$$

The seepage field equation of the fracture:

$$
d_{f} S_{f} \frac{\partial p}{\partial t}+\nabla_{\tau} \cdot\left(-d_{f} \frac{\kappa_{f}}{\eta} \nabla p\right)=-d_{f} \frac{\partial e_{r}}{\partial t}+Q_{r}
$$


TABLE 1 | Control equation parameters.

\section{Type}

Time

Flow rate

Permeability

Dynamic viscosity

Water storage coefficient

Volume strain

Seepage sink source

Crack width

Heat exchange

Crack surface normal

Derivation along crack tangent

Symbol/(Unit)
$t /(s)$
$u /(m / s)$
$\kappa /\left(m^{2}\right)$
$\eta /(P a \cdot s)$
$S /\left(P a^{-1}\right)$
$e$
$Q /\left(s^{-1}\right)$
$d_{f} /(m)$
$Q_{f}$
$n$
$\nabla_{\tau}$

$$
Q_{\mathrm{r}}=\frac{\kappa_{r}}{\eta} \frac{\partial p}{\partial n}
$$

In the formula, the subscript $f$ is expressed as fracture.

(2) The temperature field equation

\section{The Rock Temperature Field Equation:}

$$
c_{s} \rho_{s} \frac{\partial T_{S}}{\partial t}=\lambda_{s} \nabla^{2} T_{s}+W
$$

In the formula, the subscript $s$ represents rock.

Because of the low porosity, the flow rate of water in the rock is low, and the water temperature can be considered equal to the temperature of the rock, so the convection in the pores of the rock is not considered.

\section{The Fracture Water Temperature Field Equation}

$$
\begin{gathered}
d_{f} \rho_{f} c_{f} \frac{\partial T_{f}}{\partial t}+d_{f} \rho_{f} c_{f} \nabla_{\tau} T_{f}=d_{f} \nabla_{\tau} \cdot\left(\lambda_{f} \nabla_{\tau} T_{f}\right)+W_{f} \\
W_{f}=h\left(T_{s}-T_{f}\right)
\end{gathered}
$$

(3) Continuity equation

$$
\frac{\partial(\varepsilon \rho)}{\partial t}+\nabla \cdot(\rho u)=0
$$

The parameters in the formula are shown in Table 1:

\section{Coupling Relationship}

The coupling effect of the temperature field on the seepage field is mainly reflected in the change of the physical parameters of the fluid. Under the action of high temperature and high pressure, the

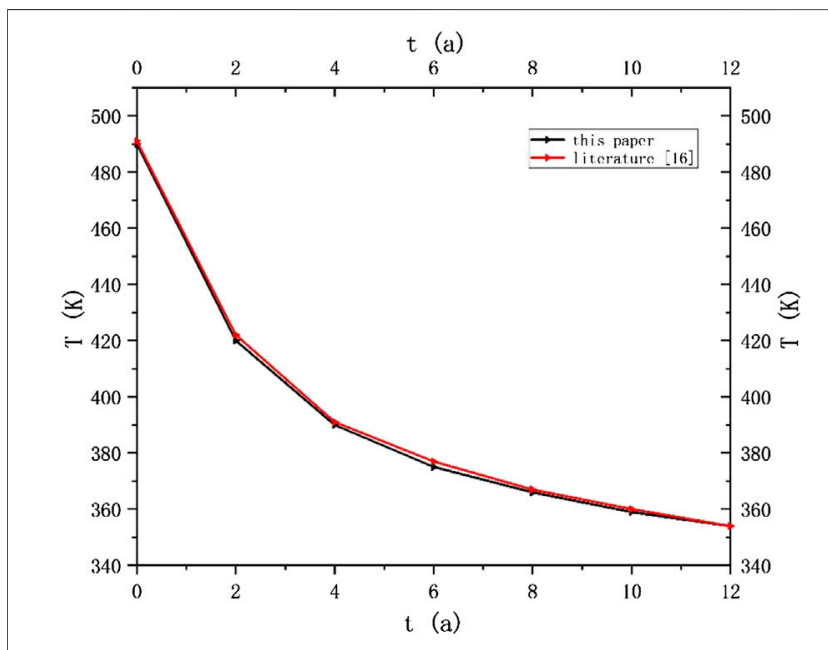

FIGURE 1 | Model verification comparison.

density of water will not be a constant, and can generally be expressed as a function of temperature and pressure (Zhao, 2010):

$$
\begin{aligned}
\frac{1}{\rho_{f}}= & 3.086-0.899017(4014.15-\mathrm{T})^{0.147166} \\
& -0.39(658.15-T)^{-1.6}(p-225.5)+\delta
\end{aligned}
$$

In the formula, $T$ represents the absolute temperature of water; $\delta$ represents the function of water temperature and pressure; $p$ represents pressure.

The influence of water temperature on the dynamic viscosity of water cannot be ignored. The dynamic viscosity of water:

$$
\eta=v \rho_{f}
$$

In the formula, $v$ represents the kinematic viscosity coefficient of water.

Both the density and viscosity of water are related to temperature, and temperature changes will directly affect the two processes of seepage and heat transfer, and their role is a strong coupling relationship. 


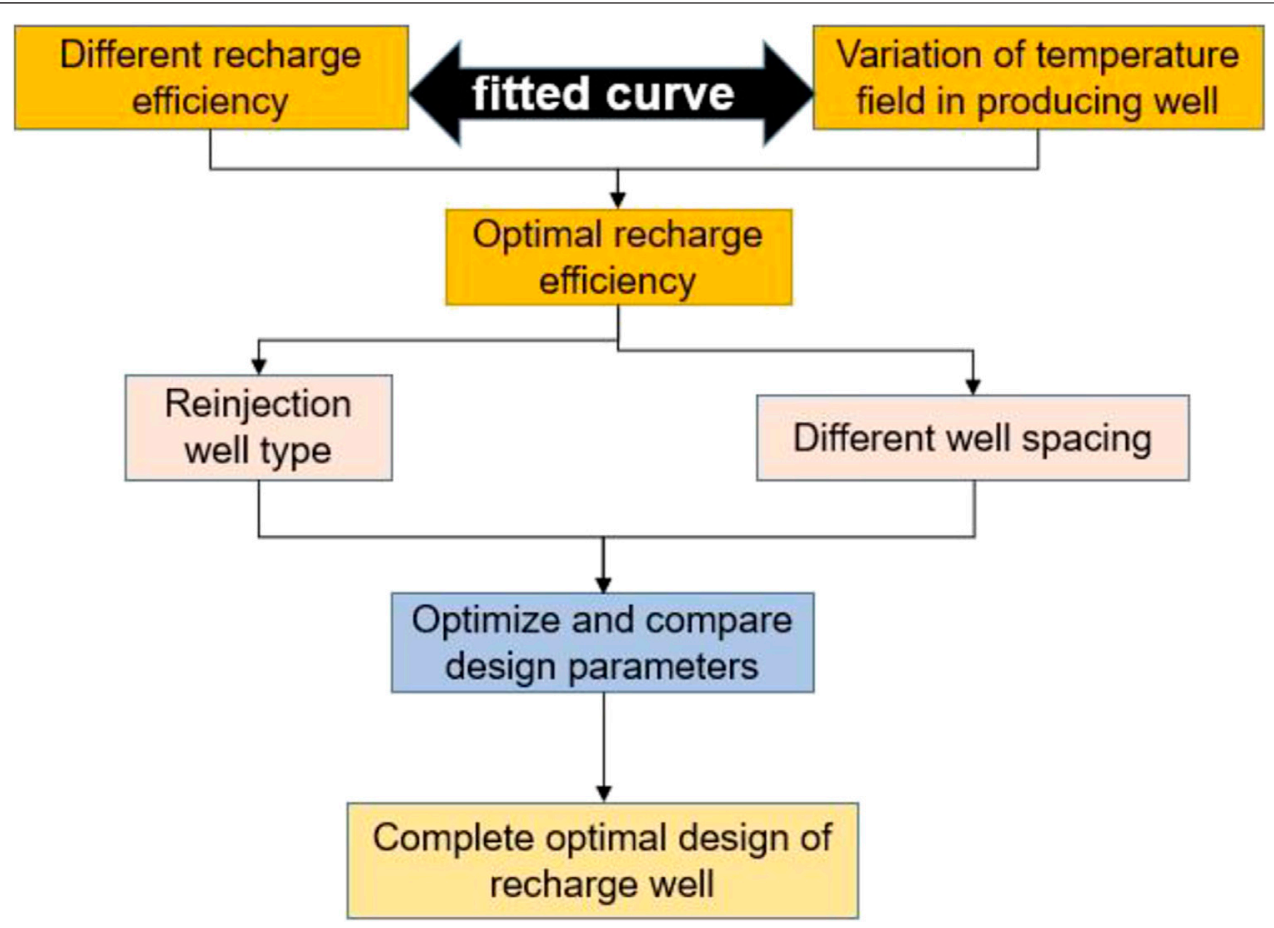

FIGURE 2 | Research ideas.

\section{Model Reliability Verification}

To verify the reliability of the model, it was applied to Tang classic THM model (the fluid heat transfer between fracture and fault is the process of heat transfer by convection and heat conduction) to calculate the output temperature (Tang et al., 2016), the model parameters are the same as those, and the calculation results are shown in Figure 1. By comparison, it is found that the calculation results in this paper are similar to the calculation results in (Tang et al., 2016), and the numerical difference is small, which, to some extent, proves the reliability of this model.

\section{Concept}

\section{(1) Thermal breakthrough}

The so-called Thermal Breakthrough refers to the production wells' pumping of groundwater and recharging of low-temperature water in the reinjection area. Due to the production wells' pumping, the low-temperature water will generate convection, which will lead to the migration of the cold water front to the hot water area at a certain speed, and eventually lead to the decrease of the temperature at the production wells. The occurrence of thermal breakthrough will reduce the operating efficiency of production well, so the occurrence of thermal breakthrough should be avoided or the time of thermal breakthrough should be delayed.

(2) "One reinjection and one production" and "One reinjection and two production"
"One reinjection and one production" means that the number of reinjection wells and production well is 1:1, and "One reinjection and two production" mean that the number of reinjection wells and production wells is 1:2. For the sake of convenience, "One reinjection and one production" and "One reinjection and two production" are used to replace these two statements.

\section{EXAMPLE MODEL}

\section{Research Methods}

This paper studies the influence of different well type designs on recharge efficiency. The calculation method of recharge efficiency is listed as follows:

$$
\gamma=\frac{Q_{1}}{Q_{2}} \cdot \frac{\eta_{2}}{\eta_{1}}
$$

In the formula, $\mathrm{Q}_{1}, \mathrm{Q}_{2}$ represents the recharge volume and pumping volume respectively, and $\eta_{1}, \eta_{2}$ represents the number of production wells and reinjection wells respectively.

The specific idea of the study is shown in Figure 2. The main method is to change the recharge efficiency, fit the relationship between different recharge efficiency and the temperature change of the production well, calculate the optimal recharge efficiency according to the fitted curve, and study under the optimal recharge efficiency. Different reinjection well types, different well spacing and different design parameters, and finally the best design plan is selected. 
TABLE 2 | Geological parameters.

\begin{tabular}{|c|c|c|c|c|}
\hline & Upper strata & Middle strata & Lower strata & Fracture \\
\hline Thermal Conductivity $(W /(m \cdot K))$ & 2 & 2 & 3.5 & 3 \\
\hline Density $\left(\mathrm{kg} / \mathrm{m}^{3}\right)$ & 1500 & 1800 & 2300 & 1200 \\
\hline Constant pressure heat capacity $(\mathrm{J} /(\mathrm{kg} \cdot \mathrm{K}))$ & 900 & 850 & 910 & 800 \\
\hline Porosity & 0.26 & 0.25 & 0.12 & 0.60 \\
\hline Permeability $\left(m^{2}\right)$ & $6.75 \times 10^{-11}$ & $6.25 \times 10-11$ & $9 \times 10^{-12}$ & \\
\hline
\end{tabular}
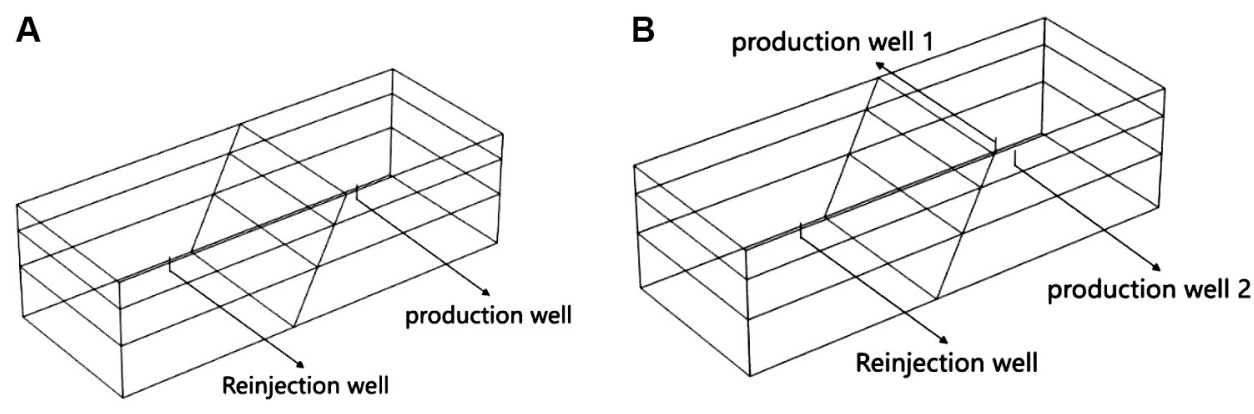

FIGURE 3 | Model diagram.

\section{Calculation Model}

Taking the geothermal exploitation and utilization development zone in North China as the research area, and based on the thermal storage depth of $1100 \mathrm{~m}$ in this area, a geothermal thermal reservoir model was established. The model includes three geological layers and a fracture surface as well as different numbers of production wells and reinjection wells.

The three geological layers and fractures have different thermal and hydraulic characteristics, and the specific parameters are shown in Table 2. The top and bottom boundaries are both defined as impervious layers. The different design methods of the two wells for irrigation and irrigation are shown in Figure 3, including one reinjection and one production (as shown in Figures 3A), one reinjection and multiple productions (as shown in Figures 3B).

The model uses a free tetrahedron for finer mesh division. The full mesh of the one reinjection and one production model contains 36,797 domain elements, 5,033 boundary elements and 426 edge elements; the full mesh of the one reinjection and multiple production model contains 37,463 domain elements, 5,067 boundary elements and 429 edge elements. The full mesh of the one reinjection and multiple production model contains 37,463 domain elements, 5,067 boundary elements, and 429 edge elements. Among them, the configuration of reinjection wells includes vertical well, inclined well, and horizontal well. The production wells are only vertical wells. The spacing between production and reinjection well includes $20,100,200,300,400,500$, and $600 \mathrm{~m}$. The pumping volume is always maintained at $5 \mathrm{~kg} / \mathrm{s}$, and the recharge volume will be changed according to a change in recharge efficiency. There are three settings for the recharge efficiency: 100,80 , and $60 \%$. The thermal breakthrough time is limited to $2 \mathrm{~K}$.

\begin{tabular}{lclc}
\hline TABLE 3 | Model parameters. & & \\
\hline Size $(\mathbf{m})$ & $\mathbf{8 0 0 * 3 0 0 * 3 0 0}$ & Surface temperature $(\mathbf{K})$ & $\mathbf{2 8 5}$ \\
\hline Upper depth $(\mathrm{m})$ & $880-930$ & $\begin{array}{l}\text { Ground ladder temperature } \\
(\mathrm{K} / \mathrm{m})\end{array}$ & 0.03 \\
& & Head $(\mathrm{mm} / \mathrm{m})$ & $1 \times 10^{-3}$ \\
Middle depth $(\mathrm{m})$ & $930-1000$ & Recharge water temperature $(\mathrm{K})$ & 280 \\
Lower depth $(\mathrm{m})$ & $1000-1100$ & $2 \times 10^{-3}$ \\
Well radius $(\mathrm{m})$ & 0.25 & Crack thickness $(\mathrm{m})$ & 910 \\
Well section length $(\mathrm{m})$ & 30 & Well depth $(\mathrm{m})$ &
\end{tabular}

\section{Parameter Setting}

The relevant parameters of the model, the thermal and hydraulic characteristics of geological layers and fractures are shown in Table 3 and Table 2 below.

\section{THE RELATIONSHIP BETWEEN RECHARGE EFFICIENCY AND TEMPERATURE FIELD CHANGES}

Figure 4 shows the temperature curves of production wells at different levels of recharge efficiency when the spacing between production and reinjection well (vertical well) is 100, 300, and $500 \mathrm{~m}$ and the spacing between production wells is $100 \mathrm{~m}$. It can be seen from Figure 4 that the temperature change curves of production well 1 and production well 2 in the case of one reinjection and two production almost overlap, so the production well in the case of one reinjection and one production and the temperature change curve of the above production well 1 are selected for analysis.

As is shown in Figure 4, the temperature decline trend is almost the same, but the decline is greater in the case of one 

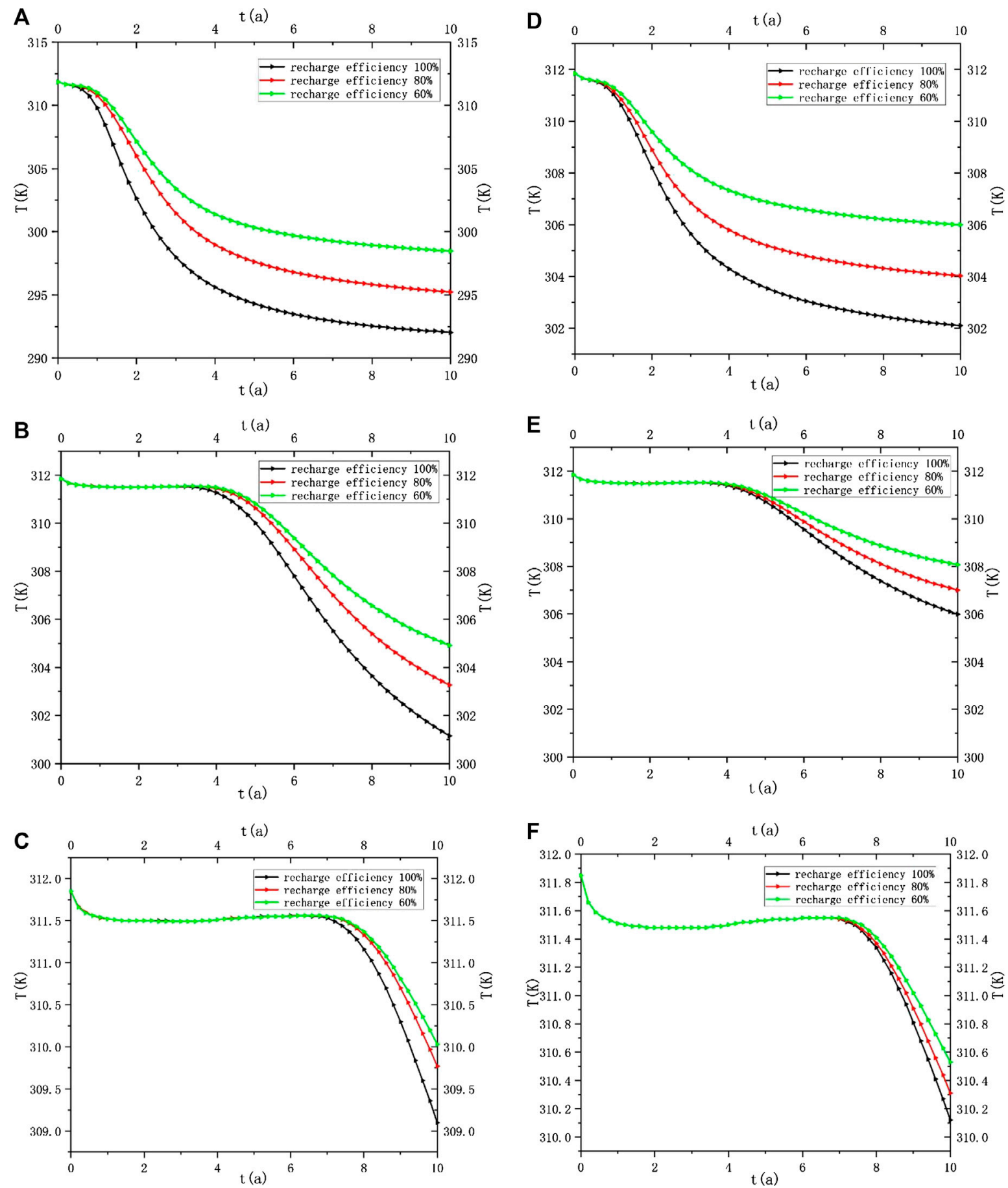

FIGURE 4 | Temperature changes of production wells under different recharge efficiency. (A) Temperature change of one reinjection and one production with a well spacing of $100 \mathrm{~m}$. (B) Temperature change of one reinjection and one production with a well spacing of $300 \mathrm{~m}$. (C) Temperature change of one reinjection and one production with a well spacing of $500 \mathrm{~m}$. (D) Temperature change of one reinjection and two production with a well spacing of $100 \mathrm{~m}$. (E) Temperature change of one reinjection and two production with a well spacing of $300 \mathrm{~m}$. (F) Temperature change of one reinjection and two production with a well spacing of $500 \mathrm{~m}$.

reinjection and one production. When the well spacing is $500 \mathrm{~m}$, the water temperature in the reinjection well has little effect on the water temperature of the production well. It took 8 years to see a significant drop in temperature and the drop was only about
$2 \mathrm{~K}$. However, with the reduction of the well spacing to $100 \mathrm{~m}$, the influence of the water temperature of the reinjection wells on the production wells became greater and greater, and even thermal breakthroughs occurred. 


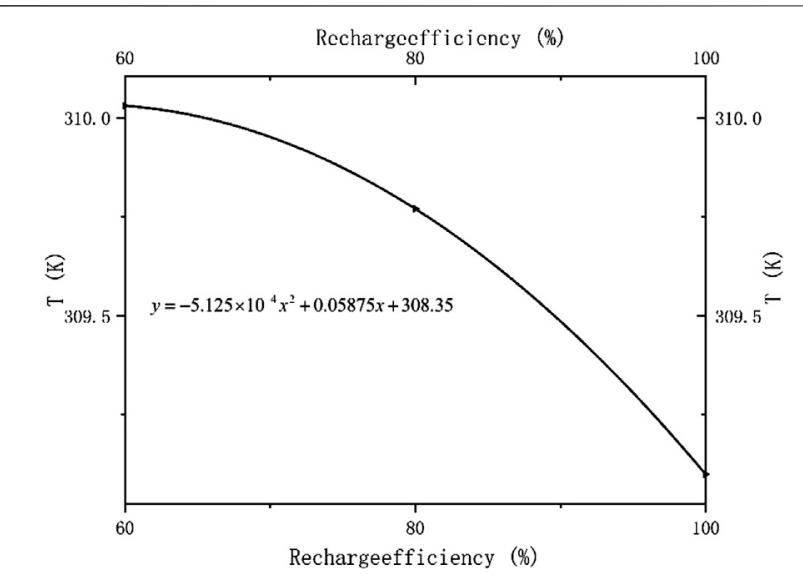

FIGURE 5 | Recharge efficiency-Temperature fitting curve.

It can be seen from Figure 4 that the temperature decrease trend of production wells is the same, but the temperature drop of production wells will decrease as the recharge efficiency increases. Compared with $60 \%$ recharge efficiency, at $100 \%$ recharge efficiency, the minimum temperature of production well 1 under the condition of one reinjection and one production is $6.44 \mathrm{~K}$ lower, and the lowest temperature of production well 1 under the condition of one reinjection and two production is also $3.91 \mathrm{~K}$ lower. This is because the setting of the recharge efficiency is determined by the ratio of recharge volume to pumping volume. In the case of one reinjection and one production, and one reinjection and two production, the lower the recharge efficiency, the lower the recharge volume. The increase in the amount of recharge will shorten the time for the cold front to reach the production well and accelerate the occurrence of thermal breakthroughs in the production well, resulting in a larger drop in its temperature. However, with the increase of the well spacing, the decrease is getting smaller and smaller.

To better characterize the relationship between recharge efficiency and temperature field, take different recharge efficiencies as the abscissa and the lowest temperature of the production well as the ordinate, and use the trend line to fit the data to get the Recharge efficiency-Temperature fitting equation. Fitting equation:

$$
y=-5.125 \times 10^{-4} x^{2}+0.05875 x+308.35
$$

Decisive factor:

$$
R^{2}=0.998
$$

Figure 5 shows the fitting curve of recharge efficiency and temperature change. The fitting equation can be used to calculate when the temperature of the production well is reduced by $2 \mathrm{~K}$. The optimal recharge efficiency that can avoid thermal breakthrough is $40.41-72.52 \%$.

The following research is based on the optimal recharge efficiency obtained by the curve and analyzes the relationship between different well spacing, well type, and temperature field
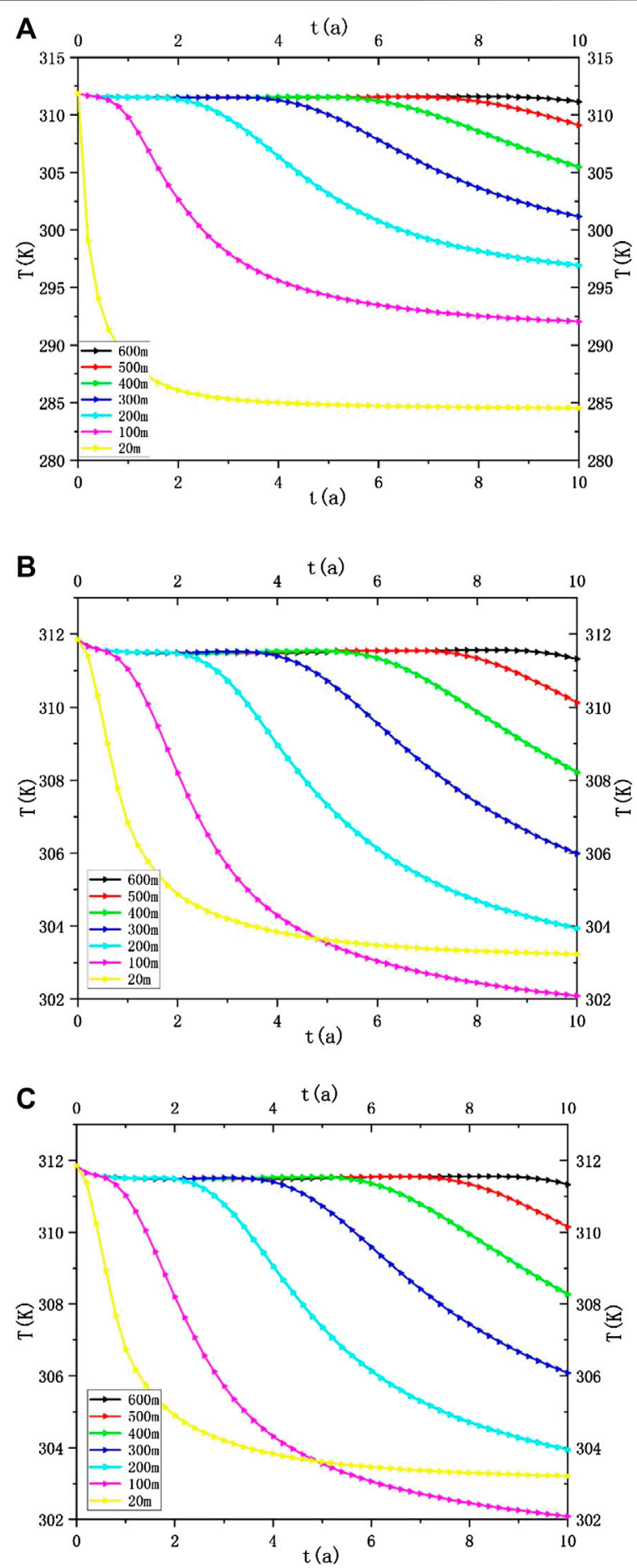

FIGURE 6 | Temperature changes of production wells at different spacings. (A) Temperature changes in the production well with one reinjection and one production. (B) Temperature changes in production well 1 with one reinjection and one production. (C) Temperature changes in production well 3 with one reinjection and one production.

and thus, obtains the well spacing between the production and reinjection well, and the well spacing between the production and production well under the optimal recharge efficiency. The best 
design plan for the well spacing between the well and the well type of the reinjection well.

\section{RESULTS AND DISCUSSION}

\section{Analysis of Temperature Field Changes in Different Well Spacing \\ Temperature Changes Under Different Well Spacings Between Production and Reinjection Wells}

In order to analyze the influence of different production and reinjection wells on the temperature field of the reservoir under different well spacings, the situation of one irrigation and one production, one irrigation and two production was simulated, and the temperature changes of production wells under different production and rejection well spacings were simulated. It is fixed as a vertical well, and the calculation result is shown in Figure 6.

It can be seen from Figure 6A that in one reinjection and one production, after the well spacing reaches $600 \mathrm{~m}$, the water temperature of the production well has a very small drop within 10 years, about $1 \mathrm{~K}$, and no thermal breakthrough occurred. When the well spacing reached $500 \mathrm{~m}$, the production well had a thermal breakthrough at 9.50 a. As shown in Figures 6B, C, in the case of one reinjection and two production, the decline of the two production well is smaller than that of one reinjection and one production, only $0.50 \mathrm{~K}$, and the curves of production well 1 and production well 2 almost overlap, with only slight differences. Thermal breakthrough did not occur when the well spacing was $500 \mathrm{~m}$, but after the well spacing was reduced to $400 \mathrm{~m}$, a thermal breakthrough occurred in 8 a. Compared with one reinjection and one production, the overall temperature change range of the production well with one reinjection and two production is relatively small, with a temperature difference ranging from 0 to $9.76 \mathrm{~K}$, while the former reaches $27.32 \mathrm{~K}$. The difference is that one reinjection and two production have the lowest temperature when the well spacing is $100 \mathrm{~m}$, while the one reinjection and one production reaches the lowest temperature when the well spacing is $20 \mathrm{~m}$.

It can be seen from Figure 6 that when the well spacing reaches $20 \mathrm{~m}$, the thermal breakthrough takes place very quickly in both cases, and both occurred within a short period of $1 \mathrm{a}$. When the well spacing is gradually reduced from 600 to $400 \mathrm{~m}$, the temperature of the production wells does not change significantly. This is because the migration resistance of the recharged water is large, and the water flow rate is slow, so interference in the production wells is small. At the same time, the larger the well spacing, the longer it takes for the recharged water to absorb the heat in the thermal reservoir during the migration process, which can fully exchange heat with geothermal fluid, and prolong the thermal breakthrough time. When the well spacing was reduced to $100 \mathrm{~m}$, the water temperature in both cases dropped significantly after $2 \mathrm{a}$. This is because the circulation channel between the recharge water and the geothermal water was greatly shortened, and the cold front reached production. The speed of the well became faster, and a thermal breakthrough occurred, resulting in a significant drop in the water temperature of the production well.
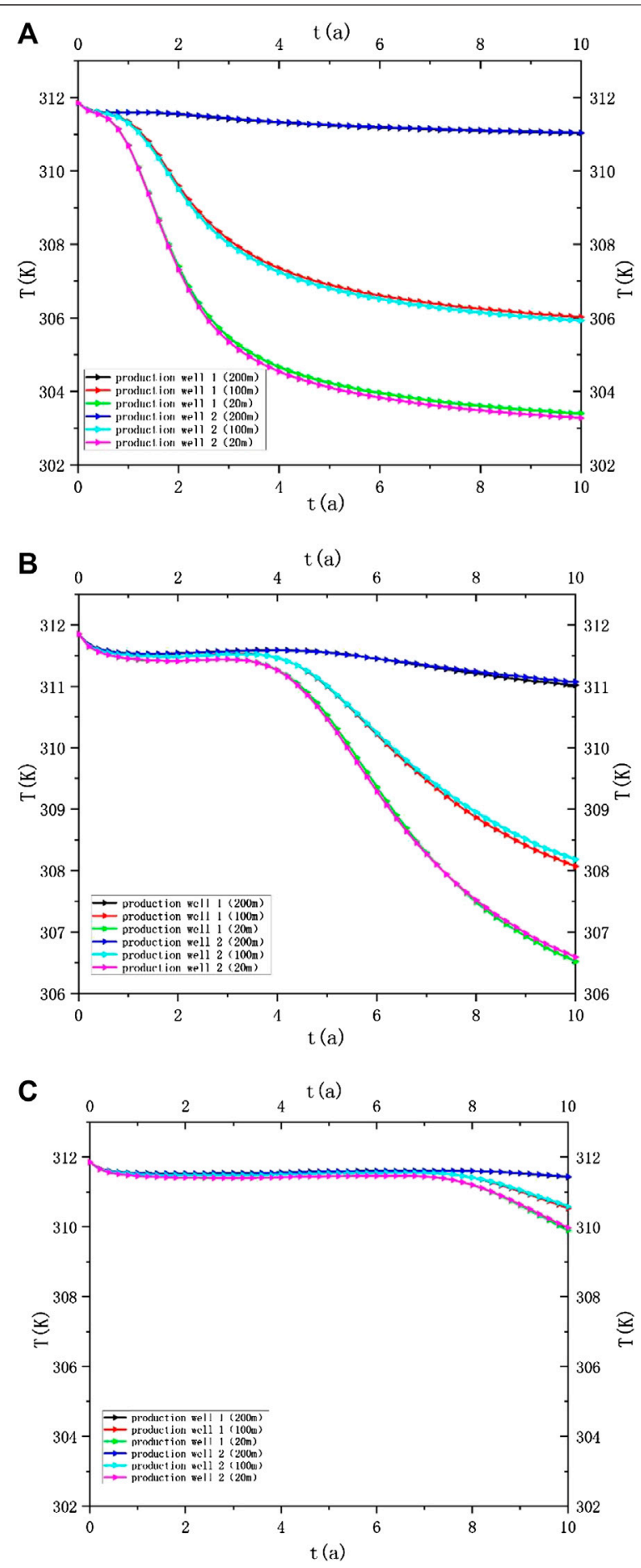

FIGURE 7 | Temperature changes of production wells 1 and 2 under different spacings between production wells. (A) Temperature changes of production well 1 and 2 at a distance of $100 \mathrm{~m}$ between production and reinjection wells. (B) Temperature changes of production well 1 and 2 at a distance of $300 \mathrm{~m}$ between production and reinjection wells. (C) Temperature changes of production well 1 and 2 at a distance of $500 \mathrm{~m}$ between production and reinjection wells. 

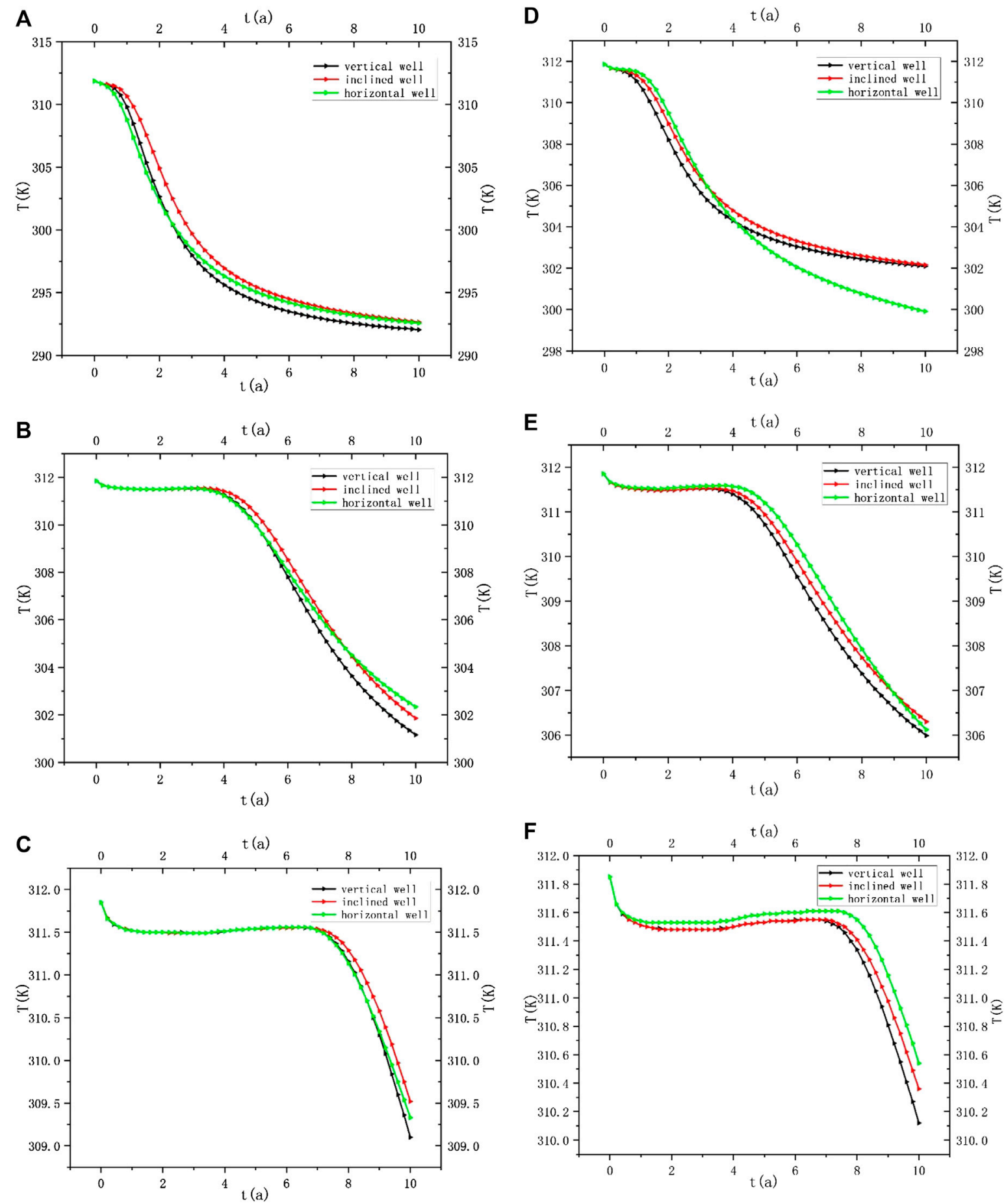

FIGURE 8 | Temperature changes of production wells under different reinjection well types. (A) Temperature change of one reinjection and one production with a well spacing of $100 \mathrm{~m}$. (B) Temperature change of one reinjection and one production with a well spacing of $300 \mathrm{~m}$. (C) Temperature change of one reinjection and one production with a well spacing of $500 \mathrm{~m}$. (D) Temperature change of one reinjection and two production with a well spacing of $100 \mathrm{~m}$. (E) Temperature change of one reinjection and two production with a well spacing of $300 \mathrm{~m}$. (F) Temperature change of one reinjection and two production with a well spacing of $500 \mathrm{~m}$. 


\section{Temperature Changes of Production Wells Under Different Spacings Between Production Wells}

Figure 7 shows the relationship between temperature changes in production wells that are caused by different spacings between production wells when the well spacing between the production and reinjection well is 100,300 , and $500 \mathrm{~m}$, and the reinjection well is a vertical well, in the case of one reinjection and two production.

It can be seen from Figure 7 that the temperature change curves of the two production wells in the case of one reinjection and two production basically overlap, and the length of the thermal breakthrough is mainly determined by the well spacing between the production and reinjection well. It can be seen that as the well spacing between the production wells increases to $500 \mathrm{~m}$, the thermal breakthrough time of the production wells is greatly delayed. However, the increase in the spacing between the production wells also causes a delay in the thermal breakthrough time of the production wells, especially after reaching $200 \mathrm{~m}$, after which point the temperature of the production wells hardly changes, and the change range is only $0.82 \mathrm{~K}$. When the spacing between production and reinjection wells is $500 \mathrm{~m}$ and the spacing between production wells is $200 \mathrm{~m}$, the temperature variation of the production wells is the smallest. This is because the time for the recharge water to move to the production well becomes longer, which leads to a longer time for its heat absorption in the thermal reservoir, and the distance between the two production wells also provides certain conditions for the warming of the recharge water.

The well spacing between production wells has no less impact on the thermal breakthrough time of production wells than the well spacing between production wells. Therefore, when designing the layout of production wells, attention should be paid to the setting of well spacing between production wells and should be maintained above $200 \mathrm{~m}$.

\section{Analysis of Temperature Field Changes of Different Reinjection Well Types}

Due to the complexity of stratum properties, there are many choices for well types of reinjection wells. To study the impact of reinjection well types on the temperature changes of a production well, we simulated the well spacing between reinjection and production well at 500,300, and $100 \mathrm{~m}$, and the spacing between production wells at $100 \mathrm{~m}$, examining different reinjection well types (vertical, $45^{\circ}$ inclined and horizontal well). The calculation results are shown in Figure $\mathbf{8}$ for the temperature changes of production wells under the vertical, $45^{\circ}$ inclined, and horizontal wells.

As shown in Figure 8, different reinjection well types have a certain impact on the temperature of the production well, and the trend of the temperature change curves of the production wells are similar. However, compared with different recharge efficiency and different well spacing, the influence law of the reinjection well type is not uniform. As shown in Figures 8A-C in the case of one reinjection and one production, the $45^{\circ}$ inclined well has the smallest impact on the temperature change of the production well under different well spacings, while Figures 8D-F show one reinjection and two production, and horizontal wells have the least impact. In short, the temperature variation range of vertical downhole production wells is the largest, and this becomes more obvious as the well spacing increases.

The three different reinjection well types have little effect on the temperature change of production wells under high well spacing because the change range is only $0.41 \mathrm{~K}$ in $10 \mathrm{a}$, and the change curves before $10 \mathrm{a}$ are almost coincident. Compared with the well spacing, the reinjection well type has a smaller effect on the temperature of the production well, but when the well spacing reaches $500 \mathrm{~m}$, the use of horizontal wells is best.

In the process of geothermal exploitation, the scheme of one reinjection and two production is adopted, and the well spacing between the reinjection and production well is maintained at more than $400 \mathrm{~m}$, and the well spacing between production wells is maintained at more than $200 \mathrm{~m}$. The use of horizontal wells for reinjection wells is more conducive to geothermal exploitation.

\section{CONCLUSION}

This comprehensive analysis examines well spacings, well types, and different well layout methods under the optimal recharge efficiency change relationship diagram. The fitting equation between the recharge efficiency and the temperature change of the production well was established by COMSOL, and an optimal recharge efficiency was obtained. The optimal recharge efficiency is determined for different well spacings between reinjection and production wells, different well types, and production well layout methods. The simulation was performed under recharge efficiency, and the following conclusions were obtained:

(1) The well spacing between the reinjection and production wells has the greatest impact on the temperature changes of the production wells. When it is gradually reduced from 600 to $400 \mathrm{~m}$, the temperature change of the production wells is not obvious, but when it is further reduced to $20 \mathrm{~m}$, a thermal breakthrough occurred at $0.20 \mathrm{a}$, and the temperature change range increased sharply. The well spacing between production wells also has a certain effect on temperature change. When the well spacing of the production well reaches $200 \mathrm{~m}$, a thermal breakthrough hardly occurs. The closer the spacing of the production well, the faster a thermal breakthrough will occur faster.

(2) Although different reinjection well types will have a certain impact on the temperature changes of production wells, as the well spacing increases, the impact will become smaller and smaller. However, the situation of one reinjection and two production is better than one reinjection and one production. Regardless of whether it is from a different well spacing or different well types, the production wells with one reinjection and one production have a faster thermal breakthrough than one reinjection and two production.

(3) Based on the above research, to improve the recharge efficiency of geothermal wells, attention should be paid to the configuration of the well spacing, as well as the number ratio of production 
wells and reinjection wells. Within the range of the optimal recharge efficiency of $40.41-72.52 \%$, the design scheme for using one reinjection two production, the well spacing between reinjection and production wells is more than $400 \mathrm{~m}$, the well spacing between production wells is more than $200 \mathrm{~m}$, and the reinjection wells are horizontal wells is optimal.

\section{DATA AVAILABILITY STATEMENT}

The original contributions presented in the study are included in the article/supplementary materials, further inquiries can be directed to the corresponding author/s.

\section{AUTHOR CONTRIBUTIONS}

$\mathrm{SD}, \mathrm{DL}$, and $\mathrm{HZ}$ participated in the design of this study. DL performed the statistical analysis and carried out the study. XS and LL collected important background information. HD provided

\section{REFERENCES}

Chávez, O., and Godínez, F. (2020). Polygeneration study of low-to-medium enthalpy geothermal reservoirs in Mexico. J. Therm. Sci. doi:10.1007/s11630020-1271-9

Daniilidis, A., Nick, H. M., and Bruhnac, D. F. (2020). Interdependencies between physical, design and operational parameters for direct use geothermal heat in faulted hydrothermal reservoirs. Geothermics 86, 101806. doi:10.1016/j. geothermics.2020.101806

Gao, X. (2018). Research on evaluation Model of pore sandstone thermal storage and recharge capacity in Tianjin. Beijing, China: China University of Geosciences.

Kong, Y., Pang, Z., Shao, H., and Kolditz, O. (2017). Optimization of well-doublet placement in geothermal reservoirs using numerical simulation and economic analysis. Environ. Earth Sci. 76, 118. doi:10.1007/s12665-017-6404-4

Liu, G., Wang, G., Zhao, Z., and Ma, F. (2020). A new well pattern of cluster-layout for deep geothermal reservoirs: case study from the Dezhou geothermal field, China. Renew. Energy. 155, 484-499. doi:10.1016/j.renene.2020.03.156

Liu, J. R. (2003). Development status of geothermal recharge. Shuiwen Dizhi Gongcheng Dizhi. 30 (3), 100-104. doi:10.3969/j.issn.1000-3665.2003.03.025

Mei, B., Guo, L., Wang, P., et al. (2018). Technology and Application progress of Geothermal well tail water recharge. CN Res Comprehensive Util. 36 (01), 168-170. doi:10.3969/j.issn.1008-9500.2018.01.061

Olasolo, P., Juárez, M. C., Morales, M. P., D'Amico, S., and Liarte, I. A. (2016). Enhanced geothermal systems (EGS): a review. Renew. Sustain. Energy Rev. 56, 133-144. doi:10.1016/j.rser.2015.11.031

Sedghi, M. M., and Zhan, H. (2020). Semi-analytical solutions of discharge variation of a qanat in an unconfined aquifer subjected to general areal recharge and nearby pumping well discharge. J. Hydrol. 584, 124691. doi:10. 1016/j.jhydrol.2020.124691

Shen, J., Wang, L., and Zhao, Y.-T. (2016). Exploration and Analysis of well formation technology for Sandstone pore type recharging Wells--A Case study assistance for data acquisition. DL drafted the manuscript. All authors read and approved the final manuscript.

\section{ACKNOWLEDGMENTS}

The authors gratefully acknowledge the financial support from: (1) Study on deep geothermal wellbore instability under large temperature difference of Changzhou Applied Basic Research Program (fund number CJ20190060); (2) The National Natural Science Foundation of Jiangsu Province under the project Study on deformation behavior and instability mechanism of surrounding rock in medium-deep geothermal drilling (fund number BK20180959); (3) State key Research and development program Unconsolidated sandstone thermal reservoir protection and efficiency drilling and completion technology and materials (fund number 2019YFB1504201).

of guantao Formation heat storage in Binhai New District, Tianjin. CN Real Estate. 21, 75-80. doi:CNKI:SUN:GFCD.0.2016-21-010

Sun, J.-P., Xu, R.-J., Jiang, Z.-B., et al. (2020). Study on single-well atmospheric recharge at confined aquifer site in Jinan. J Shandong Jianzhu Univ. 35 (03), 7-12. doi:10.12077/sdjz.2020.03.002

Tang, Z., Changhua, M., Zhang, X., et al. (2016). Numerical Simulation and Analysis of Thermal-solid flow coupling in enhanced geothermal systems . Beijing Gongye Daxue Xuebao. 42 (10), 1560-1564.

$\mathrm{Xu}, \mathrm{Y}$. (2018). Simulation and Prospect of pore-type geothermal tailwater recharge in sanqiao area, xi 'an. Xi'an, China: Chang 'an University.

Yan, F. (2020). Numerical Simulation study on improving recharge rate of porous geothermal reservoirs. Neimenggu Shiyou Huagong. 46 (02), 9-13. doi:CNKI: SUN:NMSH.0.2020-02-004

Zhao, Y. (2010). Multi-field coupling of porous media and its engineering response. Beijing, China: Science Press.

Zhu, J., Zhu, X., and Lei, H. (2012). Analysis of the influence of pressure difference compensation between geothermal recharging Wells on recharging efficiency. Acta Solar Energy. 33 (01), 56-62. doi:10.3969/j.issn.0254-0096.2012.01.010

Conflict of Interest: Author HD employed by the company Shandong Ruiheng Xingyu Petroleum Technology Development Co. Ltd.

The remaining authors declare that the research was conducted in the absence of any commercial or financial relationships that could be construed as a potential conflict of interest.

Copyright (c) 2020 Ling, Deng, Zhao, Shen, Du and Liu. This is an open-access article distributed under the terms of the Creative Commons Attribution License (CC BY). The use, distribution or reproduction in other forums is permitted, provided the original author(s) and the copyright owner(s) are credited and that the original publication in this journal is cited, in accordance with accepted academic practice. No use, distribution or reproduction is permitted which does not comply with these terms. 